\title{
ON THE EXISTENCE OF SOLUTIONS FOR NONLINEAR DIFFERENTIAL INCLUSIONS
}

\author{
BY \\ IRINA CĂPRARU and AURELIAN CERNEA*
}

\begin{abstract}
We consider a Cauchy problem for a nonlinear differential inclusion in separable and nonseparable Banach spaces under Filippov type assumptions and several existence results are obtained.

Mathematics Subject Classification 2010: 34G25.

Key words: nonlinear differential inclusion, Filippov type assumptions.
\end{abstract}

\section{Introduction}

In this paper we study nonlinear differential inclusions of the form

$$
x^{\prime} \in A x+F(t, x), \quad x(0)=x_{0},
$$

where $A$ is a $m$-dissipative operator on a Banach space, $F:[0, T] \times X \rightarrow$ $\mathcal{P}(X)$ is a set-valued map and $x_{0} \in X$.

In $[4,12]$ it is shown that FiLIPPOV's ideas ([7]) can be suitably adapted in order to prove the existence of $C^{0}$-solutions of problem (1) provided $X$ is separable. These approaches use a selection theorem due to KURATOWsKY and RYLL-NARDZEWSKI ([9]).

The aim of the present paper is twofold. On one hand, when $X$ is separable, using Bressan-Colombo theorem concerning the existence of continuous selections of lower semicontinuous multifunctions with decomposable values ([3]) we prove the existence of solutions continuously depending on a parameter for problem (1). In addition, as usual at a Filippov existence type

\footnotetext{
${ }^{*}$ Work supported by the CNCS grant PN-II-ID-PCE-2011-3-0198.
} 
theorem our result provides an estimate between the starting "quasi" solution and the solution of the differential inclusion. The result allows to obtain a continuous selection of the solution set of the problem considered. The proof follows the same pattern as in $[5,11]$ where similar results are obtained for mild solutions when $A$ is the infinitesimal generator of a strongly continuous semigroup on $X$.

On the other hand, De Blasi and Pianigiani ([6]) established the existence of mild solutions for problem (1) with $A$ the infinitesimal generator of a strongly continuous semigroup on an arbitrary, not necessarily separable Banach space $X$. Even if the ideas of Filippov are still present, the approach in $([6])$ has a fundamental difference which consists in the construction of measurable selections of the multifunction. Our second result extends the above mentioned result to the case when $A$ is an $m$-dissipative operator on $X$.

The paper is organized as follows. In section 2 we present the notations, definitions and preliminary results to be used in the sequel while in Section 3 we prove the main results.

\section{Preliminaries}

Let $X$ be a real Banach space with the norm $\|\cdot\|$. Denote by $\mathcal{P}(X)$ the family of all nonempty subsets of $X$ and by $\mathcal{B}(X)$ the family of all Borel subsets of $X$. For any subset $A \subset X$ we denote by $\operatorname{cl}(A)$ the closure of $A$.

Let $T>0, I=[0, T]$ and denote by $\mathcal{L}(I)$ the $\sigma$-algebra of all Lebesgue measurable subsets of $I$. If $A \subset I$, then $\chi_{A}(\cdot): I \rightarrow\{0,1\}$ denotes the characteristic function of $A$.

As usual, we denote by $C(I, X)$ the Banach space of all continuous functions $x(\cdot): I \rightarrow X$ endowed with the norm $|x(\cdot)|_{C}=\sup _{t \in I}\|x(t)\|$ and $L^{1}(I, X)$ the Banach space of all (Bochner) integrable functions $x(\cdot): I \rightarrow$ $X$ endowed with the norm $|x(\cdot)|_{1}=\int_{0}^{T}\|x(t)\| d t$.

For $x, y \in X$ we denote by $(x, y)=\lim _{h \uparrow 0} \frac{\|x+h y\|^{2}-\|x\|^{2}}{2 h}$ the left sided directional derivative of $\frac{1}{2}\|\cdot\|^{2}$ at $x$ in the direction $y$.

Consider $A: X \rightarrow \mathcal{P}(X)$ an operator. With $D(A)$ we denote its domain. We recall that $A$ is called dissipative if $\left(x_{1}-x_{2}, y_{1}-y_{2}\right) \leq 0$ for any $x_{1}, x_{2} \in D(A)$ and $y_{1}=A x_{1}, y_{2}=A x_{2}$.

$A$ is called $m$-dissipative if it is dissipative and $R(I-\lambda A)=X$ for any (equivalently, for some) $\lambda>0$.

Let $A: D(A) \subset X \rightarrow \mathcal{P}(X)$ be $m$-dissipative and $f(\cdot) \in L^{1}(I, X)$ which 
define the differential equation

$$
x^{\prime} \in A x+f(t) .
$$

A mapping $x(\cdot): I \rightarrow X$ is called a strong solution of $(2)$ if $x(t) \in D(A)$ a.e. on $(0, T), x(\cdot)$ is locally absolute continuous on $(0, T]$ and there exists $g \in$ $L_{l o c}^{1}((0, T], X)$ such that $g(t) \in A x(t)$ a.e. on $(0, T)$ and $x^{\prime}(t)=g(t)+f(t)$ a.e. on $(0, T)$.

It is well-known that if $X$ is reflexive then for any $x_{0} \in D(A)$ equation (2) has a unique strong solution on $I$ which satisfies $x(0)=x_{0}$ (e.g. [2]). In general, equation (2) may not have strong solutions and a way to overcome this difficulty is the concept of $C^{0}$-solutions (e.g.[10]).

Definition 1. A function $x(\cdot) \in C(I, X)$ is called a $C^{0}$-solution of $(2)$ if satisfies: for every $c \in(0, T)$ and $\varepsilon>0$ there exists

(i) $0<t_{1}<\ldots<c \leq t_{n}<T, t_{k}-t_{k-1} \leq \varepsilon, t_{0}=0$ for $k=1,2, \ldots, n$;

(ii) $f_{1}, f_{2}, \ldots, f_{n} \in X$ with

$$
\sum_{k=1}^{n} \int_{t_{k-1}}^{t_{k}}\left\|f(t)-f_{k}\right\| d t \leq \varepsilon
$$

(iii) $v_{0}, v_{1}, \ldots, v_{n} \in X$ with

$$
\frac{v_{k}-v_{k-1}}{t_{k}-t_{k-1}} \in A v_{k}+f_{k}
$$

for $k=1,2, \ldots, n$ and $\left\|x(t)-v_{k}\right\| \leq \varepsilon$ for $t \in\left[t_{k-1}, t_{k}\right), k=1,2, \ldots, n$.

According to [10], if $A$ is $m$-dissipative, $f \in L^{1}(I, X)$ and $x_{0} \in \overline{D(A)}$, there exists a unique $C^{0}$-solution of $(2)$ with $x(0)=x_{0}$.

Denote by $x\left(\cdot, x_{0}, f\right): I \rightarrow \overline{D(A)}$ the unique $C^{0}$-solution of $(2)$ which satisfies $x\left(0, x_{0}, f\right)=x_{0}$.

See [13] for the following theorem.

Theorem 2. Let $X$ be a real Banach space, let $A: D(A) \subseteq X \rightsquigarrow X$ be an $m$-dissipative operator, let $\xi, \eta \in \overline{D(A)}$ and $f, g \in L^{1}(I, X)$. Then for any $t \in I$

$$
\|x(t, \xi, f)-x(t, \eta, g)\| \leq\|\xi-\eta\|+\int_{0}^{t}\|f(s)-g(s)\| d s .
$$


See [14] for the following lemma.

Lemma 3. Let $u: I \rightarrow X$ be measurable and let $G: I \rightarrow \mathcal{P}(X)$ be a measurable closed-valued multifunction. Then, for any measurable function $v: I \rightarrow(0, \infty)$, there exists a measurable selection $g: I \rightarrow X$ of $G(\cdot)$ (i.e., such that $g(t) \in G(t)$ a.e. on $I)$ such that $\|u(t)-g(t)\|<d(u(t), G(t))+v(t)$ a.e. on $I$, where the distance between a point $x \in X$ and a subset $A \subset X$ is defined as usual by $\mathrm{d}(x, A)=\inf \{\|x-a\| ; a \in A\}$.

A subset $D \subset L^{1}(I ; X)$ is said to be decomposable if for any $u(\cdot), v(\cdot) \in D$ and any subset $A \in \mathcal{L}(I)$ one has $u \cdot \chi_{A}+v \cdot \chi_{B} \in D$, where $B=I \backslash A$. We denote by $\mathcal{D}(I, X)$ the family of all decomposable closed subsets of $L^{1}(I, X)$.

Next, $(S, d)$ is a separable metric space; we recall that a multifunction $G(\cdot): S \rightarrow \mathcal{P}(X)$ is said to be lower semicontinuous (l.s.c.) if for any closed subset $C \subset X$, the subset $\{s \in S ; G(s) \subset C\}$ is closed.

See [3] for the following two lemmas.

Lemma 4. Let $F^{*}(\cdot, \cdot): I \times S \rightarrow \mathcal{P}(X)$ be a closed valued, $\mathcal{L}(I) \otimes \mathcal{B}(S)$ measurable multifunction such that $F^{*}(t, \cdot)$ is l.s.c. for any $t \in I$. Then the multifunction $G(\cdot): S \rightarrow \mathcal{D}(I, X)$ defined by

$$
G(s)=\left\{v \in L^{1}(I, X) ; \quad v(t) \in F^{*}(t, s) \text { a.e. on } I\right\}
$$

is l.s.c. with nonempty closed values if and only if there exists a continuous mapping $\rho(\cdot): S \rightarrow L^{1}(I, X)$ such that $d\left(0, F^{*}(t, s)\right) \leq \rho(s)(t)$ a.e. on $I$, $\forall s \in S$.

Lemma 5. Let $G(\cdot): S \rightarrow \mathcal{D}(I, X)$ be a l.s.c. multifunction with closed decomposable values and let $\varphi(\cdot): S \rightarrow L^{1}(I, X), \psi(\cdot): S \rightarrow L^{1}(I, \mathbb{R})$, be continuous such that the multifunction $H(\cdot): S \rightarrow \mathcal{D}(I, X)$ defined by

$$
H(s)=c l\{v(s) \in G(s) ; \quad\|v(t)-\varphi(s)(t)\|<\psi(s)(t) \text { a.e. on } I\}
$$

has nonempty values.

Then, $H(\cdot)$ has a continuous selection, i.e. there exists a continuous mapping $h(\cdot): S \rightarrow L^{1}(I, X)$ such that $h(s) \in H(s), \forall s \in S$.

If $A, B \subset X$, the Pompeiu-Hausdorff distance between $A$ and $B$ is defined by $d_{H}(A, B)=\max \left\{d^{*}(A, B), d^{*}(B, A)\right\}$, where $d^{*}(A, B)=\sup \{d(a, B)$, $a \in A\}$. 
In what follows, $X$ is a Banach space and $Y$ is a metric space. An open (respectively, closed) ball in $Y$ with center $y$ and radius $r$ will be denoted by $B_{Y}(y, r)$ (respectively, $\left.\overline{B_{Y}(y, r)}\right)$.

A multifunction $F: Y \rightarrow \mathcal{P}(X)$ with closed bounded nonempty values is said to be $d_{H}$-continuous at $y_{0} \in Y$ if for every $\varepsilon>0$ there exists $\delta>0$ such that $d_{H}\left(F(y), F\left(y_{0}\right)\right) \leq \varepsilon$ for any $y \in B_{Y}\left(y_{0}, r\right)$. $F$ is called $d_{H}$-continuous if it is so at each point $y_{0} \in Y$.

Let $\mathcal{L}$ be the $\sigma$-algebra of the (Lebesgue) measurable sets of $\mathbb{R}$ and let $A \in \mathcal{L}$ with $\mu(A)<\infty$. A multifunction $F: Y \rightarrow \mathcal{P}(X)$ with closed bounded nonempty values is said to be Lusin measurable if for every $\varepsilon>0$ there exists a compact set $K_{\varepsilon} \subset A$, with $\mu\left(A \backslash K_{\varepsilon}\right)<\varepsilon$ such that $\left.F\right|_{K_{\varepsilon}}$ is $d_{H}$-continuous.

It is clear that if $F, G: A \rightarrow \mathcal{P}(X)$ and $f: A \rightarrow X$ are Lusin measurable, then so $F$ restricted to $B$ (measurable, $B \subset A$ ), $F+G$ and $t \mapsto d(f(t), F(t)$ ). Moreover, the uniform limit of a sequence of Lusin measurable multifunctions also is Lusin measurable.

Hypothesis H. i) $F: I \times X \rightarrow \mathcal{P}(X)$ has nonempty closed bounded values and $F(\cdot, x)$ is Lusin measurable on $I$ for any $x \in X$.

ii) There exists $L(\cdot) \in L^{1}(I,(0, \infty))$ such that $\forall t \in I$

$$
d_{H}\left(F\left(t, x_{1}\right), F\left(t, x_{2}\right)\right) \leq L(t)\left\|x_{1}-x_{2}\right\|, \quad \forall x_{1}, x_{2} \in X .
$$

iii) There exists $q(\cdot) \in L^{1}(I,(0, \infty))$ such that $\forall t \in I$ we have $F(t, 0) \subset$ $q(t) B(0,1)$.

See [6] for the following lemma.

Lemma 6. i) Let $F_{i}: I \rightarrow \mathcal{P}(X), i=1,2$, be two Lusin measurable multifunctions and let $\varepsilon_{i}>0, i=1,2$, be such that $H(t):=\left(F_{1}(t)+\varepsilon_{1} B\right) \cap$ $\left(F_{2}(t)+\varepsilon_{2} B\right) \neq \emptyset, \forall t \in I$. Then the multifunction $H: I \rightarrow \mathcal{P}(X)$ has a Lusin measurable selection $h: I \rightarrow X$.

ii) Assume that Hypothesis $H$ is satisfied. Then, for any $x(\cdot): I \rightarrow X$ continuous, $u(\cdot): I \rightarrow X$ measurable and $\varepsilon>0$

a) the multifunction $t \rightarrow F(t, x(t))$ is Lusin measurable on $I$,

b) the multifunction $G: I \rightarrow \mathcal{P}(X)$ defined by $G(t):=(F(t, x(t))+\varepsilon B) \cap$ $B_{X}(u(t), d(u(t), F(t, x(t)))+\varepsilon)$ has a Lusin measurable selection $g: I \rightarrow X$.

In what follows we are concerned with the following Cauchy problem

$$
x^{\prime} \in A x+F(t, x), \quad x(0)=x_{0},
$$


where $A$ is an $m$-dissipative operator on the separable Banach space $X$, $F: I \times X \rightarrow \mathcal{P}(X)$ and $x_{0} \in X$.

Definition 7. A continuous mapping $x: I \rightarrow \overline{D(A)}$ is said to be a $C^{0}$ solution of problem (4) if $x(0)=x_{0}$ and there exists $f(\cdot) \in L^{1}(I, X)$ with $f(t) \in F(t, x(t))$ a.e. on $I$ and $x(\cdot)$ is a $C^{0}$-solution on $I$ of the equation (2) in the sense of Definition 1.

We shall call $(x(\cdot), f(\cdot))$ a trajectory-selection pair of $(4)$ if $f(t) \in F(t, x(t))$ a.e. on $I$ and $x(\cdot)$ is a $C^{0}$-solution of (4).

We shall use the following notation for the solution sets of (4)

$$
\mathcal{S}\left(x_{0}\right)=\left\{x(\cdot) ; \quad x(\cdot) \quad \text { is a } \mathrm{C}^{0}-\text { solution of }(4)\right\} .
$$

Definition 8. If $X$ is not separable, a continuous mapping $x: I \rightarrow$ $\overline{D(A)}$ is said to be a $C^{0}$-solution of problem (4) if $x(0)=x_{0}$ and there exists a Lusin measurable function $f(\cdot) \in L^{1}(I, X)$ with $f(t) \in F(t, x(t))$ a.e. on $I$ and $x(\cdot)$ is a $C^{0}$-solution on $I$ of the equation (2) in the sense of Definition 1.

\section{Main results}

In order to establish our continuous version of Filippov theorem for the problem (4) we need the following hypotheses.

Hypothesis 3.1. i) $F: I \times X \rightarrow \mathcal{P}(X)$ has nonempty closed values and is $\mathcal{L}(I) \otimes \mathcal{B}(X)$ measurable;

ii) there exists $L(\cdot) \in L^{1}\left(I, \mathbb{R}_{+}\right)$such that for almost all $t \in I, F(t, \cdot)$ is $L(t)$ - Lipschitz in the sense that $d_{H}(F(t, x), F(t, y)) \leq L(t)\|x-y\|, \forall x, y \in$ $X$.

Let $A: D(A) \subset X \rightarrow \mathcal{P}(X)$ be an $m$-dissipative operator.

Hypothesis 3.2. i) $S$ is a separable metric space and $a(\cdot): S \rightarrow X$, $c(\cdot): S \rightarrow(0, \infty)$ are continuous mappings;

ii) there exist the continuous mappings $g(\cdot): S \rightarrow L^{1}(I, X), y(\cdot): S \rightarrow$ $C(I, X)$ and $\rho(\cdot): S \rightarrow L^{1}\left(I, \mathbb{R}_{+}\right)$such that $y(s)(\cdot)$ is a $C^{0}$-solution of $x^{\prime} \in A x+g(s)(t), \forall s \in S$ and $d(g(s)(t), F(t, y(s)(t))) \leq \rho(s)(t)$ a.e. on $I$, $\forall s \in S$. 
Next we use the following notations

$$
\begin{aligned}
& m(t)=\int_{0}^{t} L(u) d u \\
& \xi(s)(t)=e^{m(t)}(T c(s)+\|a(s)-y(s)(t)\|)+\int_{0}^{T} \rho(s)(u) e^{m(t)-m(u)} d u .
\end{aligned}
$$

Theorem 9. Assume that Hypotheses 3.1 and 3.2 are satisfied. Then there exist the continuous mappings $x(\cdot): S \rightarrow C(I, X)$ and $f(\cdot): S \rightarrow$ $L^{1}\left(I, \mathbb{R}_{+}\right)$such that for any $s \in S,(x(s)(\cdot), f(s)(\cdot))$ is a trajectory-selection pair of $x^{\prime} \in A x+F(t, x), x(0)=a(s)$ and

$$
\begin{aligned}
& \|x(s)(t)-y(s)(t)\| \leq \xi(s)(t), \quad \forall t \in I, s \in S, \\
& \|f(s)(t)-g(s)(t)\| \leq L(t) \xi(s)(t)+\rho(s)(t)+c(s) \quad \text { a.e. on } I, \forall s \in S .
\end{aligned}
$$

Proof. We make the following notations:

$$
\begin{aligned}
& \varepsilon_{n}(s)=c(s) \frac{n+1}{n+2}, n=0,1, \ldots, \quad d(s)=\|a(s)-y(s)(0)\|, \\
& \rho_{n}(s)(t)=\int_{0}^{t} \rho(s)(u) \frac{(m(t)-m(u))^{n-1}}{(n-1) !} d u+\frac{(m(t))^{n-1}}{(n-1) !}\left(T \varepsilon_{n}(s)+d(s)\right), n \geq 1 .
\end{aligned}
$$

Set also $x_{0}(s)(t)=y(s)(t), \forall s \in S, t \in I$.

We consider the multifunctions $G_{0}(\cdot), H_{0}(\cdot)$ defined, respectively, by

$$
\begin{aligned}
& G_{0}(s)=\left\{v \in L^{1}(I, X) ; \quad v(t) \in F(t, y(s)(t)) \text { a.e. on } I\right\}, \\
& H_{0}(s)=c l\left\{v \in G_{0}(s) ; \quad\|v(t)-g(s)(t)\|<\rho(s)(t)+\varepsilon_{0}(s)\right\} .
\end{aligned}
$$

Since $d(g(s)(t), F(t, y(s)(t))) \leq \rho(s)(t)<\rho(s)(t)+\varepsilon_{0}(s)$, according to Lemma 3 , the set $H_{0}(s)$ is not empty.

Set $F_{0}^{*}(t, s)=F(t, y(s)(t))$. Note that $d\left(0, F_{0}^{*}(t, s)\right) \leq\|g(s)(t)\|+\rho(s, t)=$ $\rho^{*}(s)(t)$ and $\rho^{*}(\cdot): S \rightarrow L^{1}(I, X)$ is continuous.

Applying Lemma 4 and Lemma 5 we obtain the existence of a continuous solution $f_{0}(\cdot)$ of $H_{0}(\cdot)$, i.e.

$$
f_{0}(s)(t) \in F(t, y(s)(t)) \quad \text { a.e. on } I, \forall s \in S
$$

and $\left\|f_{0}(s)(t)-g(s)(t)\right\| \leq \rho_{0}(s)(t)=\rho(s)(t)+\varepsilon_{0}(s), \forall s \in S, t \in I$. 
Let $x_{1}(s)(\cdot)$ be the unique $C^{0}$-solution of the problem $x^{\prime}=A x+f_{0}(s)(t)$, $x(0)=a(s)$ and, by Theorem 2 , we have

$$
\begin{aligned}
\left\|x_{1}(s)(t)-x_{0}(s)(t)\right\| & \leq\|a(s)-y(s)(0)\|+\int_{0}^{t}\left\|f_{0}(s)(u)-g(s)(u)\right\| d u \\
& \leq d(s)+\int_{0}^{t} \rho_{0}(s)(u) d u<\rho_{1}(s)(t) .
\end{aligned}
$$

We shall construct, using the same idea as in $[5,11]$, two sequences of approximations $f_{n}(\cdot): S \rightarrow L^{1}(I, X), x_{n}(\cdot): S \rightarrow C(I, X)$ with the following properties:

a) $f_{n}(\cdot): S \rightarrow L^{1}(I, X), x_{n}(\cdot): S \rightarrow C(I, X)$ are continuous;

b) $f_{n}(s)(t) \in F\left(t, x_{n}(s)(t)\right)$ a.e. on $I, s \in S$;

c) $\left\|f_{n}(s)(t)-f_{n+1}(s)(t)\right\| \leq L(t) \rho_{n}(s)(t)$ a.e. on $I, s \in S$;

d) $x_{n+1}(s)(\cdot)$ is the unique $C^{0}$-solution of the problem $x^{\prime}=A x+$ $f_{n}(s)(t), x(0)=a(s)$. Suppose we have already constructed $f_{i}(\cdot), x_{i}(\cdot)$ satisfying a)-c) and let $x_{n+1}(\cdot)$ be as in $\mathrm{d}$ ). From c) and Theorem 2 we have

$$
\begin{aligned}
\left\|x_{n+1}(s)(t)-x_{n}(s)(t)\right\| & \leq \int_{0}^{t}\left\|f_{n}(s)(u)-f_{n-1}(s)(u)\right\| d u \\
& \leq \int_{0}^{t} L(u) \rho_{n}(s)(u) d u<\rho_{n+1}(s)(t) .
\end{aligned}
$$

On the other hand,

$$
\begin{aligned}
d\left(f_{n}(s)(t), F\left(t, x_{n+1}(s)(t)\right)\right) & \leq L(t)\left\|x_{n+1}(s)(t)-x_{n}(s)(t)\right\| \\
& <L(t) \rho_{n+1}(s)(t)
\end{aligned}
$$

To prove that $H_{n+1}(s)$ is not empty, we note first that the real function $t \rightarrow r_{n}(s)(t)=c(s) \frac{T L(t) m(t)^{n}}{(n+2)(n+3) n !}$ is measurable and strictly positive for any $s \in S$. Using (8) we get $d\left(f_{n}(s)(t), F\left(t, x_{n+1}(s)(t)\right)\right) \leq L(t) \| x_{n+1}(s)(t)-$ $x_{n}(s)(t) \|-r_{n}(s)(t) \leq L(t) \rho_{n+1}(s)(t)$ and, therefore, according to Lemma 4 , there exists $v(\cdot) \in L^{1}(I, X)$ such that $v(t) \in F\left(t, x_{n}(s)(t)\right)$ a.e. on $I$ and $\left\|v(t)-f_{n}(s)(t)\right\|<d\left(f_{n}(s)(t), F\left(t, x_{n}(s)(t)\right)\right)+r_{n}(s)(t)$ and hence $H_{n+1}(s)$ is not empty.

Set $F_{n+1}^{*}=F\left(t, x_{n+1}(s)(t)\right)$ and note that we may write $d\left(0, F_{n+1}^{*}(t, s)\right) \leq$ $L(t)\left\|x_{n+1}(s)(t)-x_{n}(s)(t)\right\| \leq\left\|f_{n}(s)(t)\right\|+L(t) \rho_{n+1}(s)(t)=\rho_{n+1}^{*}(s)(t)$ a.e. on $I$ and $\rho_{n+1}^{*}(\cdot): S \rightarrow L^{1}(I, X)$ is continuous. 
By Lemmas 4 and 5 there exists a continuous mapping $f_{n+1}(\cdot): S \rightarrow$ $L^{1}(I, X)$ such that

$$
\begin{aligned}
& f_{n+1}(s)(t) \in F\left(t, x_{n+1}(s)(t)\right) \quad \text { a.e. on } I, \forall s \in S, \\
& \left\|f_{n+1}(s)(t)-f_{n}(s)(t)\right\| \leq L(t) \rho_{n+1}(s)(t) \quad \text { a.e. on } I, \forall s \in S .
\end{aligned}
$$

From (7) we obtain

$$
\begin{aligned}
\left\|x_{n+1}(s)(\cdot)-x_{n}(s)(\cdot)\right\|_{C} & \leq\left\|f_{n+1}(s)(\cdot)-f_{n}(s)(\cdot)\right\|_{1} \\
& \leq \frac{(m(T))^{n}}{n !}\left(\|\rho(s)(\cdot)\|_{1}+T c(s)+d(s)\right) .
\end{aligned}
$$

Therefore, $f_{n}(s)(\cdot), x_{n}(s)(\cdot)$ are Cauchy sequences in the Banach space $L^{1}(I, X)$ and $C(I, X)$ respectively. Let $f(\cdot): S \rightarrow L^{1}(I, X)$ and $x(\cdot)$ : $S \rightarrow C(I, X)$ be their limits. The function $s \rightarrow\|\rho(s)(\cdot)\|_{1}+T(s)+d(s)$ is continuous, hence locally bounded. Therefore (9) implies that for every $s^{\prime} \in S$ the sequence $f_{n}\left(s^{\prime}\right)(\cdot)$ satisfies the Cauchy condition uniformly with respect to $s^{\prime}$ on some neighbourhood of $s$. Hence $s \rightarrow f(s)(\cdot)$ is continuous from $S$ into $L^{1}(I, X)$.

From (9), as before, $x_{n}(s)(\cdot)$ is Cauchy in $C(I, X)$ locally uniformly with respect to $s$. So, $s \rightarrow x(s)(\cdot)$ is continuous from $S$ into $C(I, X)$. On the other hand, since $x_{n}(s)(\cdot)$ converges uniformly to $x(s)(\cdot)$ and

$$
d\left(f_{n}(s)(t), F(t, x(s)(t))\right) \leq L(t)\left\|f_{n}(s)(t)-x(s)(t)\right\|
$$

a.e. on $I, \forall s \in S$. Passing to the limit along a subsequence of $f_{n}(\cdot)$ converging pointwise to $f(\cdot)$ we obtain

$$
f(s)(t) \in F(t, x(s)(t)) \quad \text { a.e. on } I, \forall s \in S .
$$

Let $x^{*}(s)(\cdot)$ be the unique $C^{0}$-solution of the Cauchy problem $x^{\prime} \in$ $A x+f(s)(t), x(0)=a(s)$. Since $\left\|x_{n+1}(s)(t)-x^{*}(s)(t)\right\| \leq \int_{0}^{t} \| f_{n}(s)(u)-$ $f(s)(u) \| d u$, letting $n \rightarrow \infty$ we get $x^{*}(s)(\cdot) \equiv x(s)(\cdot)$.

By adding inequalities c) for all $n$ and using the fact that $\sum_{i \geq 1} \rho_{i}(s)(t) \leq$ $\xi(s)(t)$ we obtain

$$
\begin{array}{r}
\left\|f_{n+1}(s)(t)-g(s)(t)\right\| \leq \sum_{l=0}^{n}\left\|f_{l+1}(s)(t)-f_{l}(s)(u)\right\|+\left\|f_{l}(s)(u)-g(s)(t)\right\| \\
(10) \leq \sum_{l=0}^{n} L(t) \rho_{l+1}(s)(t)+\rho(s)(t)+\varepsilon_{0}(s) \leq L(t) \xi(s)(t)+\rho(s)(t)+c(s) .
\end{array}
$$


Similarly, by adding (7) we get

$$
\left\|x_{n+1}(s)(t)-y(s)(t)\right\| \leq \sum_{l=0}^{n} \rho_{l}(s)(t) \leq \xi(s)(t) .
$$

By passing to the limit in (10) and (11) we obtain (5) and (6), respectively. The proof is complete.

Theorem 9 allows to obtain the next corollary which is a general result concerning continuous solutions of the solution set of (4).

Hypothesis 3.3. Hypothesis 3.1 is satisfied and there exists $\rho_{0}(\cdot) \in$ $L^{1}\left(I, \mathbb{R}_{+}\right)$such that $d(0, F(t, 0)) \leq \rho_{0}(t)$ a.e. on $I$.

Theorem 10. Assume that Hypothesis 3.3 is satisfied. Then there exists a function $x(\cdot, \cdot): I \times X \rightarrow X$ such that:

a) $x(\cdot, \xi) \in \mathcal{S}(\xi), \forall \xi \in X$

b) $\xi \rightarrow x(\cdot, \xi)$ is continuous from $X$ into $C(I, X)$.

Proof. We take $S=X, a(\xi)=\xi, \forall \xi \in X, c(\cdot): X \rightarrow(0, \infty)$ an arbitrary function, $g(\cdot)=0, y(\cdot)=0, \rho(\xi)(t)=\rho_{0}(t), \forall x \in X, t \in I$, and we apply Theorem 9 in order to obtain the conclusion of the theorem.

Remark 11. A similar result as in the Theorem 10 is obtained in [11] in the particular case when $X$ is a Hilbert space and $A=-B$ with $B$ maximal monotone. Moreover, the result in Theorem 10 may be obtained as a particular case of the main result (Theorem 9) in [1]. The result in [1] is obtained for a more general problem, namely a Volterra integral solution. We underline the fact that our Theorem 9 cannot be obtained from any of the results in [1].

We consider now the case when $X$ is not separable.

Theorem 12. We assume that Hypothesis $H$ is satisfied. Then for any $x_{0} \in X$ there exists $x(\cdot): I \rightarrow X$ a $C^{0}$-solution of problem (4).

Proof. Let us first note that, if $z(\cdot): I \rightarrow X$ is continuous, then every Lusin measurable selection $u: I \rightarrow X$ of the multifunction $t \mapsto$ $F(t, z(t))+B(0,1)$ is Bochner integrable on $I$. More precisely, for any $t \in I$ we have $\|u(t)\| \leq d_{H}(F(t, z(t))+B(0,1), 0) \leq d_{H}(F(t, z(t)), F(t, 0))+$ $d_{H}(F(t, 0), 0)+1 \leq L(t)\|z(t)\|+q(t)+1$. Let $0<\varepsilon<1, \varepsilon_{n}=\frac{\varepsilon}{2^{n+2}}$. 
Consider an arbitrary Lusin measurable function $f_{0}(\cdot): I \rightarrow X$, that is Bochner integrable and define $x_{0}(\cdot)$ the unique $C^{0}$-solution of the problem $x^{\prime} \in A x+f_{0}(t), x(0)=x_{0}$. Since $x_{0}(\cdot)$ is continuous, by Lemma 6 ii) there exists a Lusin measurable function $f_{1}(\cdot): I \rightarrow X$ satisfying $f_{1}(t) \in$ $\left(F\left(t, x_{0}(t)\right)+\varepsilon_{1} B(0,1)\right) \cap B\left(f_{0}(t), d\left(f_{0}(t), F\left(t, x_{0}(t)\right)\right)+\varepsilon_{1}\right), t \in I$.

Obviously, $f_{1}(\cdot)$ is Bochner integrable on $I$. Define $x_{1}(\cdot)$ the unique $C^{0}$-solution of the problem $x^{\prime} \in A x+f_{1}(t), x(0)=x_{0}$. We construct, by induction, a sequence $x_{n}(\cdot): I \rightarrow X, n \geq 2$, with $x_{n}(\cdot)$ the unique $C^{0}$ solution of the problem $x^{\prime} \in A x+f_{n}(t), x(0)=x_{0}$, where $f_{n}(\cdot): I \rightarrow X$ is a Lusin measurable function satisfying

$$
\begin{aligned}
& f_{n}(t) \in\left(F\left(t, x_{n-1}(t)\right)+\varepsilon_{n} B\right) \cap B\left(f_{n-1}(t),\right. \\
&\left.d\left(f_{n-1}(t), F\left(t, x_{n-1}(t)\right)\right)+\varepsilon_{n}\right), \quad t \in I .
\end{aligned}
$$

At the same time, as we saw at the beginning of the proof, $f_{n}(\cdot)$ is also Bochner integrable. From (12), for $n \geq 2$ and $t \in I$ we have

$$
\begin{aligned}
& \left\|f_{n}(t)-f_{n-1}(t)\right\| \leq d\left(f_{n-1}(t), F\left(t, x_{n-1}(t)\right)\right)+\varepsilon_{n} \leq d\left(f_{n-1}(t), F\left(t, x_{n-2}(t)\right)\right) \\
& +d_{H}\left(F\left(t, x_{n-2}(t)\right), F\left(t, x_{n-1}(t)\right)\right)+\varepsilon_{n} \leq \varepsilon_{n-1}+L(t)\left\|x_{n-1}-x_{n-2}\right\|+\varepsilon_{n} .
\end{aligned}
$$

Since $\varepsilon_{n-1}+\varepsilon_{n}<\varepsilon_{n-2}$, we deduce that

$$
\left\|f_{n}(t)-f_{n-1}(t)\right\| \leq \varepsilon_{n-2}+L(t)\left\|x_{n-1}(t)-x_{n-2}(t)\right\|, n \geq 2 .
$$

Denote $m(t)=\int_{0}^{t} L(u) d u$ and $\rho_{0}(t)=d\left(f_{0}(t), F\left(t, x_{0}(t)\right)\right), t \in I$. Next, we prove by recurrence that for $n \geq 2$ and $t \in I$ we have

$$
\begin{aligned}
& \left\|x_{n}(t)-x_{n-1}(t)\right\| \leq \sum_{k=0}^{n-2} \int_{0}^{t} \varepsilon_{n-2-k} \frac{(m(t)-m(u))^{k}}{k !} d u \\
& +\varepsilon_{0} \int_{0}^{t} \frac{(m(t)-m(u))^{n-1}}{(n-1) !} d u+\int_{0}^{t} \frac{(m(t)-m(u))^{n-1}}{(n-1) !} \rho_{0}(t) d u .
\end{aligned}
$$

We start with $n=2$. By (12), (13) and (3) for $t \in I$, we have

$$
\begin{aligned}
& \left\|x_{2}(t)-x_{1}(t)\right\| \leq \int_{0}^{t}\left\|f_{2}(s)-f_{1}(s)\right\| d s \leq \int_{0}^{t}\left[\varepsilon_{0}+L(s)\left\|x_{1}(s)-x_{0}(s)\right\|\right] d s \\
& \leq \varepsilon_{0} t+\int_{0}^{t} L(s) \int_{0}^{s}\left\|f_{1}(u)-f_{0}(u)\right\| d u d s \leq \varepsilon_{0} t+\int_{0}^{t} L(s) \int_{0}^{s}\left(\rho_{0}(u)+\varepsilon_{1}\right) d u d s \\
& \leq \varepsilon_{0} t+\int_{0}^{t}\left(\rho_{0}(u)+\varepsilon_{1}\right) \int_{0}^{t} L(s) d s d u=\varepsilon_{0} t+\int_{0}^{t}(m(t)-m(s))\left(\rho_{0}(s)+\varepsilon_{0}\right) d s,
\end{aligned}
$$


i.e. (14) is satisfied for $n=2$. Using again (12), (13) and (3) we have

$$
\begin{aligned}
& \left\|x_{n+1}(t)-x_{n}(t)\right\| \leq \int_{0}^{t}\left\|f_{n+1}(u)-f_{n}(u)\right\| d u \\
& \leq \int_{0}^{t}\left[\varepsilon_{n-1}+L(s)\left\|x_{n}(s)-x_{n-1}(s)\right\|\right] d s \\
& \leq \varepsilon_{n-1} t+\int_{0}^{t} L(s)\left[\sum_{k=0}^{n-2} \int_{0}^{s} \varepsilon_{n-2-k} \frac{(m(s)-m(u))^{k}}{k !} d u\right. \\
& \left.+\int_{0}^{s} \frac{(m(s)-m(u))^{n-1}}{(n-1) !}\left(\rho_{0}(u)+\varepsilon_{0}\right) d u\right] d s \\
& =\varepsilon_{n-1} t+\sum_{k=0}^{n-2} \varepsilon_{n-2-k} \int_{0}^{t}\left[\int_{0}^{s} \frac{(m(s)-m(u))^{k}}{k !} L(s) d u\right] d s \\
& +\int_{0}^{t} L(s)\left(\int_{0}^{s} \frac{(m(s)-m(u))^{n-1}}{(n-1)) !} L(s)\left[\rho_{0}(u)+\varepsilon_{0}\right] d u\right) d s \\
& =\varepsilon_{n-1} t+\sum_{k=0}^{n-2} \varepsilon_{n-2-k} \int_{0}^{t}\left[\int_{u}^{t} \frac{(m(s)-m(u))^{k}}{k !} L(s) d s\right] d u \\
& +\int_{0}^{t}\left[\int_{u}^{t} \frac{(m(s)-m(u))^{n-1}}{(n-1)) !} L(s) d s\right]\left[\rho_{0}(u)+\varepsilon_{0}\right] d u \\
& =\varepsilon_{n-1} t+\sum_{k=0}^{n-2} \varepsilon_{n-2-k} \int_{0}^{t} \frac{(m(s)-m(u))^{k+1}}{(k+1) !} d u \\
& +\int_{0}^{t} \frac{(m(s)-m(u))^{k}}{k !}\left[\rho_{0}(u)+\varepsilon_{0}\right] d u \\
& =\sum_{k=0}^{n-1} \varepsilon_{n-1-k} \int_{0}^{t} \frac{(m(s)-m(u))^{k}}{k !} d u \\
& +\int_{0}^{t} \frac{(m(s)-m(u))^{n}}{n !}\left[\rho_{0}(u)+\varepsilon_{0}\right] d u,
\end{aligned}
$$

so that (14) holds true for $n+1$.

It follows from (14) that for $n \geq 2$ and $t \in I$ we have

$$
\left\|x_{n}(t)-x_{n-1}(t)\right\| \leq a_{n}
$$


where

$$
a_{n}=\sum_{k=0}^{n-2} \varepsilon_{n-2-k} \frac{m(t)^{k}}{k !}+\frac{m(t)^{n-1}}{(n-1) !}\left[\int_{0}^{T} \rho_{0}(u) d u+\varepsilon_{0}\right] .
$$

Obviously, the series with $n$-th term $a_{n}$ is convergent.

So, from (15) we have that $x_{n}(\cdot)$ converges uniformly on $I$ to a continuous function $x(\cdot): I \rightarrow X$.

On the other hand, by (13) we have $\left\|f_{n}(t)-f_{n-1}(t)\right\| \leq \varepsilon_{n-2}+L(t) a_{n-1}$, $t \in I, n \geq 3$, which implies that the sequence $f_{n}(\cdot)$ converges to a Lusin measurable function $f(\cdot): I \rightarrow X$. Since $x_{n}(\cdot)$ is bounded and $\left\|f_{n}(t)\right\| \leq$ $L(t)\left\|x_{n-1}(t)\right\|+q(t)+1$ we deduce that $f(\cdot)$ is also Bochner integrable.

Let $x^{*}(\cdot): I \rightarrow X$ be the unique $C^{0}$-solution of the Cauchy problem $x^{\prime} \in A x+f(t), \quad x(0)=x_{0}$. By $(3)\left\|x_{n}(t)-x^{*}(t)\right\| \leq \int_{0}^{t}\left\|f_{n}(s)-f(s)\right\| d s$ and using the Lebesgue dominated convergence theorem we obtain $x(\cdot)=x^{*}(\cdot)$. On the other hand, from (12) we get $f_{n}(t) \in F\left(t, x_{n}(t)\right)+\varepsilon_{n} B(0,1), t \in$ $I, n \geq 1$ and letting $n \rightarrow \infty$ we have $f(t) \in F(t, x(t)), t \in I$ and the proof is complete.

\section{REFERENCES}

1. Aizicovici, S.; Staicu, V. - Continuous selections of solution sets to Volterra integral inclusions in Banach spaces, Electron. J. Differential Equations, 2006, 11 pp. (electronic).

2. BARBu, V. - Nonlinear Semigroups and Differential Equations in Banach Spaces, Institutul European, Iaşi, Romania, 2011.

3. Bressan, A.; Colombo, G. - Extensions and selections of maps with decomposable values, Studia Math., 90 (1988), 69-86.

4. C̆̆PRARU, I. - A Filippov-type theorem for nonlinear differential inclusions, Proc. Int. Stud. Conf. Pure Appl. Math., "Al. I. Cuza" Univ., Iaşi, 55-62, 2011.

5. Cernea, A. - Continuous version of Filippov's theorem for a semilinear differential inclusion, Stud. Cerc. Mat., 49 (1997), 319-330.

6. DE Blasi, F.S.; PiAnigiani, G. - Evolution inclusions in non-separable Banach spaces, Comment. Math. Univ. Carolin., 40 (1999), 227-250.

7. Filippov, A.F. - Classical solutions of differential equations with multi-valued righthand side, SIAM J. Control, 5 (1967), 609-621.

8. Frankowska, H. - A priori estimates for operational differential inclusions, J. Differential Equations, 84 (1990), 100-128. 
9. Kuratowski, K.; Ryll-Nardzewski, C. - A general theorem on selectors, Bull. Acad. Polon. Sci. Sér. Sci. Math. Astronom. Phys., 13 (1965), 397-403.

10. Lakshmikantham, V.; Leela, S. - Nonlinear Differential Equations in Abstract Spaces, International Series in Nonlinear Mathematics: Theory, Methods and Applications, 2, Pergamon Press, Oxford-New York, 1981.

11. Staicu, V. - Continuous selections of solution sets to evolution equations, Proc. Amer. Math. Soc., 113 (1991), 403-413.

12. Tolstonogov, A.A. - Properties of integral solutions of differential inclusions with m-accretive operators, (Russian) Mat. Zametki, 49 (1991), 119-131, 159, translation in Math. Notes, 49 (1991), 636-644.

13. VRabie, I.I. - Compactness Methods for Nonlinear Evolutions, With a foreword by A. Pazy. Second edition, Pitman Monographs and Surveys in Pure and Applied Mathematics, 75, Longman Scientific \& Technical, Harlow, copublished in the United States with John Wiley \& Sons, Inc., New York, 1995.

14. ZHU, Q.J. - On the solution set of differential inclusions in Banach space, J. Differential Equations, 93 (1991), 213-237.

Received: 20.XI.2012

Accepted: $19 . I V .2013$
Faculty of Mathematics, "Al. I. Cuza" University of Iaşi, Bd. Carol I 11, 700506 Iaşi, ROMANIA

irina_capraru@yahoo.com

Faculty of Mathematics and Informatics, University of Bucharest, Academiei 14, 010014 Bucharest, ROMANIA acernea@fmi.unibuc.ro 